\title{
FUNCTIONS OF BROWNIAN MOTION
}

\author{
JOHN B. WALSH
}

ABSTRACT. The class of continuous functions $h$ for which $h$ (Brownian motion) is a Markov process is determined.

Let $B$ be a one-dimensional Brownian motion. For what continuous real valued functions $h$ is $\left\{h\left(B_{t}\right), t \geq 0\right\}$ again a Markov process? If we require that $h\left(B_{t}\right)$ be again a Brownian motion, Dudley [1] has shown that $h$ must be either $x$ or $-x$; there should be a larger class of functions which transform Brownian motion into a Markov process. What is perhaps surprising is that the class is still relatively small.

To be definite, suppose $B$ is defined canonically on the space $\Omega$ of all continuous real valued functions on $[0, \infty)$, i.e. $B=\left(\Omega, F_{t}, B_{t}, P^{x}\right)$, where $B_{t}$ is the canonical process, $\left.F_{t}=\sigma B_{s}, s \leq t\right\}$, and $P^{x}$ is the distribution of Brownian motion with initial value $x$. When we say $h\left(B_{t}\right)$ is a Markov process, we mean that there is a transition function $Q(t, x, A)$ on $h(\mathbf{R})$ such that for any initial distribution, $\left\{h\left(B_{t}\right), t \geq 0\right\}$ is a Markov process with transition function $Q$. We let $\mathcal{H}$ be the class of all continuous functions with this property.

There is a general criterion, due to Rosenblatt for Markov chains [4] (see also [2, p. 325]). A continuous function $h$ is in $\mathcal{H}$ iff for all $x$ and $y, h(x)=h(y)$ implies that $P_{t}\left(x, h^{-1}(A)\right)=P_{t}\left(y, h^{-1}(A)\right)$ for all $t \geq 0$ and Borel sets $A$. Equivalently,

(1) $h \in \mathcal{H}$ iff $h(x)=h(y)$ implies that the process $\left\{h\left(B_{t}\right), t \geq 0\right\}$ has the same distribution under $P^{y}$ as under $P^{x}$.

It is clear that $\mathcal{H}$ contains constants and strictly monotone functions. What is more interesting is that the remainder of $\mathcal{H}$ is composed of two classes of functions:

(I) functions $h$ having a unique maximum or minimum, say at $x_{0}$. Then $h$ is symmetric about $x_{0}$ and strictly monotone on either side;

(II) nonconstant functions $h$ having more than one local extremum. In

Received by the editors September 5, 1973 and, in revised form, February 4, 1974.

AMS (MOS) subject classifications (1970). Primary 60 J60, 60 J65. 
this case $h$ is periodic, symmetric about any local extremum, and strictly monotone between adjacent maxima and minima.

There is a more compact description of $\mathcal{H}$. Let

$$
\begin{aligned}
& f_{1}(x) \equiv 0, \quad f_{2}(x)=x, \\
& f_{3}(x)=|x|, \quad f_{4}(x)=d(x, 2 \mathrm{~N}),
\end{aligned}
$$

i.e. $f_{4}(x)$ is the distance from $x$ to the nearest even integer.

Theorem. $\mathcal{H}$ is the class of functions $h$ of the form

$$
h(x)=g \circ f_{i}(a x+b), \quad 1 \leq i \leq 4,
$$

where $a$ and $b$ are constants and $g$ is continuous and strictly monotone.

We can dispatch three-quarters of the theorem directly. If $h$ is of the form (2), $h\left(B_{t}\right)$ is a Markov process. Indeed, $f_{3}\left(B_{t}\right)$ is reflecting Brownian motion and $f_{4}\left(B_{t}\right)$ is Brownian motion on $[0,1]$, reflecting at both endpoints, while a strictly monotone (hence one-to-one) function of a Markov process is again a Markov process. Conversely, constants and strictly monotone functions can certainly be written in the form (2). It remains to deal with functions which are neither constant nor strictly monotone-and which are consequently not one-to-one.

Lemma 1. Let $h \in \mathcal{H}$. Then any local maximum (resp. minimum) of $h$ is also a global maximum (resp. minimum). If $h$ is constant on any interval, it is a constant function. by (1)

Proof. Suppose $h\left(x_{0}\right)=b$, a local maximum of $h$. If $h(x)=h\left(x_{0}\right)$, then

$$
\begin{aligned}
P^{x}\left\{h\left(B_{t}\right)\right. & \leq b \text { for all small enough } t\} \\
& =P^{x}\left\{h\left(B_{t}\right) \leq b \text { for all small enough } t\right\}=1 .
\end{aligned}
$$

This implies that $x$ is also a local maximum of $h$. As $h$ is continuous, we can conclude $h(x) \leq b$ for all $x$. Finally, if $h$ is constant on some interval, it has points which are simultaneously local (hence global) maxima and minima. Thus inf $h=\sup h$, and $h$ is constant. Q.E.D.

A consequence of Lemma 1 is that if $h \in \mathcal{H}$ is nonconstant and has no global extremum in some interval, it is strictly monotone there.

Define $A_{a}=\{x: h(x)=a\}$, and let $d\left(x, A_{a}\right)$ be the distance from $x$ to $A_{a}$. 
Lemma 2. Let $h \in \mathcal{H}$. If $A_{a} \neq \varnothing$, there is a function $u$ on $h(\mathbf{R})$ such that $d\left(x, A_{a}\right)=u \circ h(x)$ for all $x$.

Proof. Suppose $h(x)=h\left(x^{\prime}\right)$ and let $I$ and $I^{\prime}$ be the maximal open subintervals of $\mathbf{R}-A_{a}$ which contain $x$ and $x^{\prime}$ respectively. Write $I=$ $(x-c, x+d)$ and $I^{\prime}=\left(x^{\prime}-c^{\prime}, x^{\prime}+d^{\prime}\right)$. We define $T_{a}=\inf \left\{t: h\left(B_{t}\right)=a\right\}$. If $B_{0}=x$, then $T_{a}=\inf \left\{t: B_{t}-B_{0}=-c\right.$ or $\left.d\right\}$, and if $B_{0}=x^{\prime}$, then $T_{a}=$ inf $\left\{t: B_{t}-B_{0}=-c^{\prime} \text { or } d^{\prime}\right\}^{\text {. Since }} h(x)=h\left(x^{\prime}\right), T_{a}$ has the same distribu tion under $P^{x}$ as under $P^{x^{\prime}}$. By Lemma 3 below, we conclude that

$$
d\left(x, A_{a}\right)=\min (c, d)=\min \left(c^{\prime}, d^{\prime}\right)=d\left(x^{\prime}, A_{a}\right) .
$$

Thus we can set $u(h(x))=\min (c, d)$. This proves the lemma.

Lemma 3. Let $0<c \leq d$ be (possibly infinite) reals, and let $T=$ $\inf \left\{t: B_{t}=-c\right.$ or $\left.d\right\}$. Suppose $B_{0}=0$. Then both $c$ and $d$ are determined by the distribution of $T$.

Proof. First, $c=d=\infty$ iff $P^{0}\{T=\infty\}=1$, so we may as well suppose $c<\infty$. For the moment, we also suppose $d<\infty$. Let

$$
f_{a}(x)=E^{x}\left\{e^{-\alpha T}\right\}, \quad-c \leq x \leq d .
$$

Then $[3, \mathrm{p} .129] f_{\alpha}$ satisfies

$$
1 / 2 f_{\alpha}^{\prime \prime}=\alpha f_{\alpha}, \quad f_{\alpha}(-c)=f_{\alpha}(d)=1 .
$$

Solving this and setting $x=0$ :

$$
f_{a}(0)=(\sinh \sqrt{2 \alpha} c+\sinh \sqrt{2 \alpha} d) / \sinh \sqrt{2 \alpha}(c+d)
$$

Now the distribution of $T$ determines $f_{\alpha}(0)$, and this in turn determines both $c$ and $d$. Indeed

$$
\begin{aligned}
& c=-\lim _{\alpha \rightarrow \infty}(2 \alpha)^{-1 / 2} \log f_{\alpha}(0), \\
& d=-\lim _{\alpha \rightarrow \infty}(2 \alpha)^{-1 / 2} \log \left[f_{\alpha}(0)-\exp (-\sqrt{2 \alpha} c)\right] .
\end{aligned}
$$

In fact, (5) holds even if $d=\infty$, for then the condition $f_{\alpha}(d)=1$ is replaced by $0 \leq f_{\alpha} \leq 1$, and we get $f_{\alpha}(0)=\exp (-\sqrt{2 \alpha} c)$.

We can now complete the proof of the Theorem. Suppose $h \in \mathcal{H}$ has a single local (hence global) extremum at $x_{0}$. We may as well suppose $x_{0}$ is a minimum. Let $a=h\left(x_{0}\right)$. By Lemma 2 applied to $A_{a}=\left\{x_{0}\right\}$ :

$$
\left|x-x_{0}\right|=u \circ h(x) \text {. }
$$


But $h$ is continuous and strictly monotone on $\left(-\infty, x_{0}\right]$ and on $\left[x_{0}, \infty\right)$ (for it is nowhere constant) so that $u$ must also be continuous and strictly increasing on the range of $h$. In particular, it is invertible, so (6) gives us

$$
h(x)=u^{-1} \circ f_{3}\left(x-x_{0}\right) .
$$

This leaves the case where $b$ is nonconstant and has more than one local extremum. Let $a=\inf b<\sup h=b$. Noting that there is a local (hence global) minimum between any two local maxima, we may assume there exist $x_{0}$ and $x_{1}$ such that $h\left(x_{0}\right)=a, h\left(x_{1}\right)=b$, and $h$ is strictly monotone between $x_{0}$ and $x_{1}$. To fix our ideas, suppose $x_{0}<x_{1}$. By Lemma 2

$$
d\left(x, A_{a}\right)=u \circ h(x) .
$$

Consider $A_{a} \cdot A_{a} \neq\left\{x_{0}\right\}$, for, as $x_{1}$ is a strict local maximum, we can find $z$ and $z^{\prime}$ in some neighborhood $\left(x_{1}-\epsilon, x_{1}+\epsilon\right)$ such that $x_{0}<z<x_{1}<z^{\prime}$ and $h(z)=h\left(z^{\prime}\right)$. Then $d\left(z^{\prime}, A_{a}\right)=d\left(z, A_{a}\right)$, which requires a point $x^{\prime}>$ $x_{1}$ in $A_{a}$. Evidently, $x^{\prime}=z^{\prime}+\left(z-x_{0}\right)$. Letting $\epsilon \rightarrow 0$ we see that $x^{\prime}=x_{0}$ $+d$, where $d=2\left|x_{1}-x_{0}\right|$. Similarly, $x_{1}-d \in A_{b}$. Repeating this argument, one sees that

$$
A_{a}=\left\{x_{0} \pm n d, n=0,1,2, \cdots\right\}, \quad A_{b}=\left\{x_{1} \pm n d, n=0,1,2, \ldots\right\} .
$$

Thus

$$
d\left(x, A_{a}\right)=f_{4}\left(\left(x-x_{0}\right) / d\right) .
$$

But both $h(x)$ and $f_{4}\left(\left(x-x_{0}\right) / d\right)$ are strictly monotone on $x_{0}, x_{1}$, hence $u$ is strictly monotone on $[a, b]$, the range of $h$. In particular, $u^{-1}$ exists, and (8) and (9) give

$$
h(x)=u^{-1} \circ f_{4}\left(\left(x-x_{0}\right) / d\right),
$$

which completes the proof.

One can ask the more general question: "What Borel measurable functions $h$ transform Brownian motion into a strong Markov process?" The function $h$ may no longer have local maxima, but Lemma 2 remains valid (the continuity of $b$ did not enter into its proof), and one can use it and arguments similar to those in its proof to determine the sets $A_{a}$ directly. For example, if $A_{a}$ is a singleton for all $a, b$ must be one-to-one. If $A_{a}=$ $\left\{x_{1}, x_{2}\right\}$ for some $a$, one argues using Lemma 2 that if $x_{0}=\left(x_{1}+x_{2}\right) / 2$, 
then $A_{b\left(x_{0}\right)}=\left\{x_{0}\right\}$, and that for any other $c$ for which $A_{c}$ is nonempty, $A_{c}=$ $\left\{x_{0}-y, x_{0}+y\right\}$ for some $y>0$. This would imply that $h$ is of the form $g \circ f_{3}\left(x-x_{0}\right)$, where $g$ is one to-one. Similar arguments can be used to establish that if $A_{a}$ contains more than two points, its elements form an arithmetic progression.

Thus no new ideas are involved in this more general question, but the details are both more complicated and less interesting than before. The answer is not, however. If we add the function $f_{5}(x)=x-[x]$ to our list, then if $h$ is a real valued Borel measurable function, $h\left(B_{t}\right)$ will be a strong Markov process iff $h(x)=g \circ f_{i}(a x+b), 1 \leq i \leq 5$, where $a$ and $b$ are constants and $g$ is one-to-one Borel measurable.

\section{REFERENCES}

1. R. M. Dudley, Non-linear equivalence transformations of Brownian motion, Z. Wahrscheinlichkeitstheorie und Verw. Gebiete 20 (1971), 249-258. MR 46 \#6485.

2. E. B. Dynkin, Markov processes, Fizmatgiz, Moscow, 1963; English transl., Vols. I, II, Die Grundlehren der math. Wissenschaften, Bände 121, 122, Academic Press, New York; Springer-Verlag, Berlin, 1965. MR 33 \# 1886; \# 1887.

3. K. Itô and H. P. McKean, Diffusion processes and their sample paths, Die Grundlehren der math. Wissenschaften, Band 125, Academic Press, New York; Springer-Verlag, Berlin, 1965. MR 33 \#8031.

4. M. Rosenblatt, Functions of a Markov process that are Markovian, J. Math. Mech. 8 (1959), 665-681.

DEPARTMENT OF MATHEMATICS, UNIVERSITY OF BRITISH COLUMBIA, VANCOUVER 8, BRITISH COLUMBIA, CANADA 